\title{
ON FIXED POINTS OF AUTOMORPHISMS OF NON-ORIENTABLE UNBORDERED KLEIN SURFACES
}

\author{
Grzegorz GromadzKi
}

\begin{abstract}
In 1973, Macbeath found a general formula for the number of points fixed by an arbitrary orientation preserving automorphism of a Riemann surface $X$. It was given in terms of a group $G$ of conformal automorphisms of $X$ and the ramification data of the covering $X \rightarrow X / G$, which corresponds to the so called universal covering transformation group. In these terms, for the case of a cyclic group of automorphisms of an unbordered non-orientable Klein surface, the formula was given later by Izquierdo and Singerman and here we find formulas valid for an arbitrary (finite) group $G$ of automorphisms.
\end{abstract}

\section{Introduction}

It is not difficult to see, that the set of fixed points of an automorphism of a compact Riemann surface consists either of isolated points or simple closed Jordan curves called ovals (and sometimes mirrors). The last case occurs only for anticonformal involutions, in the literature known as symmetries. In 1973, Macbeath found in [14] a formula for the number of points fixed by an arbitrary automorphism of a Riemann surface $X$ in terms of the group of conformal automorphisms of $X$ and the ramification data of its action, which corresponds to the so called universal covering transformation group. Later we found (in $[\mathbf{7}]$ - see also $[8]$ ) a similar formula for the number of ovals of a symmetry.

It is worth mentioning here that the possible number of ovals of a symmetry of a Riemann surface is given by the classical Harnack-Weichold Theorem [10], [17]. A useful role is also played by the method of HoareSingerman [11], concerning non-normal subgroups of NEC-groups, which

2000 Mathematics Subject Classification. Primary: 30F; Secondary: $14 \mathrm{H}$.

Key words. Automorphisms of Riemann and Klein surfaces, fixed-point set, Fuchsian and NEC-groups, uniformization.

Supported by SAB2005-0049 and by MTM2005-01637 of the Spanish Ministry of Sciences and Education. 
allowed the relations between the total number of ovals of two symmetries and the order of their product to be found in [4], and the nature of the set of fixed points of involutions of compact Klein surfaces with boundary in [3].

The case of non-orientable surfaces is essentially different, since both types of fixed points may occur simultaneously. For a cyclic group $G$ of automorphisms, Izquierdo and Singerman found [12] formulas for the number of isolated fixed points and the number of ovals of any automorphisms from $G$, in terms of the universal covering transformation group. Here we find such formulas for an arbitrary group of automorphisms of such a surface. We also give some illustrative examples.

\section{Preliminaries}

We shall use combinatorial methods based on the Riemann uniformization theorem and theory of Fuchsian and NEC-groups as in [5], where the reader can find necessary concepts and facts together with the precise references to the original sources. By an unbordered nonorientable Klein surface we mean a non-orientable compact topological surface with a dianalytic structure which, roughly speaking, differs from the classical analytic one by the fact, that the complex conjugation is allowed for transition functions of charts, see [1] for preciseness. The principal role in combinatorial study of such surfaces is nowadays being played by the counterpart of the Riemann uniformization theorem, by which such a surface $X$ can be represented as the orbit space $\mathcal{H} / \Gamma$ of the hyperbolic plane, with respect to the action of some, so called surface $N E C$-group [1]. The notion of a Klein surface has already been known to Klein himself, but until seventies such surfaces have been studied either as algebraic curves with real equations or as Riemann surfaces together with a single antiholomorphic involution by considering automorphisms commuting with it. By [1] , for a surface given as such orbit space, its group of automorphisms $G$ can be represented as the factor group $\Lambda / \Gamma$ for some other NEC-group $\Lambda$ and the pair $(\mathcal{H}, \Lambda)$ is called universal covering transformation group for the action $(G, X)$.

An NEC-group is a discrete subgroup of the group of isometries $\mathcal{G}$ of the hyperbolic plane $\mathcal{H}$, including those reversing orientation with a compact orbit space. If $\Lambda$ contains no orientation preserving elements of finite order, then it is called surface NEC-group. Using fundamental regions, Macbeath and Wilkie [13], [18] associated to every NEC-group $\Lambda$ the so called signature, which determines its algebraic structure. It has 
the form

$$
\left(g ; \pm ;\left[m_{1}, \ldots, m_{r}\right] ;\left\{\left(n_{11}, \ldots, n_{1 s_{1}}\right), \ldots,\left(n_{k 1}, \ldots, n_{k s_{k}}\right)\right\}\right)
$$

and mentioned above surface groups have signatures $(g ; \pm ;[-] ;\{-\})$. The numbers $m_{i} \geq 2$ are called the proper periods, the brackets $\left(n_{i 1}, \ldots, n_{i s_{i}}\right)$, the period cycles, the numbers $n_{i j} \geq 2$ are the link periods and $g \geq 0$ is said to be the orbit genus of $\Lambda$. The orbit space $\mathcal{H} / \Lambda$ is a surface with $k$ boundary components, orientable or not according to the sign being + or - , having topological genus $g$ and the canonical projection $\mathcal{H} \rightarrow \mathcal{H} / \Lambda$ is a covering ramified over $r$ interior points with ramification indices $m_{i}$ and over $s_{i}$ points lying on each boundary component with ramification indices $n_{i j}$. A group with the signature (1) has a presentation given by generators:
(a) $x_{i}, \quad i=1, \ldots, r$
(elliptic elements)
(b) $c_{i j}, \quad i=1, \ldots, k, j=0, \ldots, s_{i}$,
(hyperbolic reflections)
(c) $e_{i}, \quad i=1, \ldots, k$,
(boundary generators)
(d) $a_{i}, b_{i}, \quad i=1, \ldots, g$ if the sign is,+ (hyperbolic translations)
$d_{i}, \quad i=1, \ldots, g$ if the sign is,$- \quad$ (glide reflections)

and relations

(1) $x_{i}^{m_{i}}=1$,

$$
\begin{aligned}
& i=1, \ldots, r, \\
& i=1, \ldots, k, \\
& i=1, \ldots, k, j=0, \ldots, s_{i}, \\
& i=1, \ldots, k, j=1, \ldots, s_{i},
\end{aligned}
$$

(2) $c_{i s_{i}}=e_{i}^{-1} c_{i 0} e_{i}$,

(3) $c_{i j}^{2}=1$,

$\left(c_{i j-1} c_{i j}\right)^{n_{i j}}=1$,

(4) $x_{1} \ldots x_{r} e_{1} \ldots e_{k} a_{1} b_{1} a_{1}^{-1} b_{1}^{-1} \ldots a_{g} b_{g} a_{g}^{-1} b_{g}^{-1}=1$ or

$x_{1} \ldots x_{r} e_{1} \ldots e_{k} d_{i}^{2} \ldots d_{g}^{2}=1$.

Any system of generators of an NEC-group, which satisfies the above relations, will be called a canonical system of generators and it is known, that every element of finite order in $\Lambda$ is conjugate either to a canonical reflection or to a power of some canonical elliptic element $x_{i}$ or else to a power of the product of two consecutive canonical reflections $c_{i j-1}, c_{i j}$.

For every NEC-group we have an associated fundamental region, whose hyperbolic area $\mu(\Lambda)$ depends only on the group and for a group with signature (1) it is given by

(2) $2 \pi\left(\varepsilon g+k-2+\sum_{i=1}^{r}\left(1-1 / m_{i}\right)+1 / 2 \sum_{i=1}^{k} \sum_{i=1}^{s_{i}}\left(1-1 / n_{i j}\right)\right)$, 
where $\varepsilon=2$ or 1 according to the sign being + or - . It is known that an abstract group with the presentation given by the above generators and relations can be realized as an NEC-group with the signature (1) if and only if (2) is positive. Finally, if $\Lambda^{\prime}$ is a subgroup of finite index in an NEC-group $\Lambda$, then it is an NEC-group itself and the Hurwitz-Riemann formula (known also as the Hurwitz formula) says that

$$
\left[\Lambda: \Lambda^{\prime}\right]=\mu\left(\Lambda^{\prime}\right) / \mu(\Lambda) .
$$

\section{On fixed points of an automorphism of unbordered non-orientable Klein surfaces}

Let $X=\mathcal{H} / \Gamma$ be a non-orientable, unbordered Klein surface, where $\Gamma$ is an NEC-group with signature $(g ;-;[-] ;\{-\})$ and let the action of $G$ on $X$ be defined by an epimorphism $\theta: \Lambda \rightarrow G$, where $\Lambda$ is an NEC-group with signature (1). We start this principal section of the paper with the lemma describing the nature of the set of points fixed by an automorphism of $X$.

Lemma 3.1. The set of points fixed by an automorphism of an unbordered, non-orientable Klein surface consists of isolated points and ovals.

Proof: The canonical projection $\pi: \mathcal{H} \rightarrow X$ is a non-ramified covering and the action of $G$ on $X$ is defined by

$$
g x=\pi(\lambda h) \text { if } g=\theta(\lambda), x=\pi(h) .
$$

So if $\varphi=\theta(\lambda)$, then $x=\pi(h)$ is its fixed point if and only if $\lambda h=\gamma h$ for some $\gamma \in \Gamma$, which means that $\gamma^{-1} \lambda$ fixes $h$. But then $\gamma^{-1} \lambda$ is either an elliptic element or a reflection, say $c$, since these are the only hyperbolic isometries with fixed points. In the first case $x$ is obviously an isolated fixed point. Now, if in the second case $\ell$ is the axis of $c$, then on the one hand $\pi(\ell)$ is the fixed-point set of $\varphi$, while on the other hand it is homeomorphic to a circle, as $\pi$ is an unramified covering.

Theorem 3.2. The number of isolated fixed points of $\varphi \in G$ on $X$ is given by the formula

$$
\left|\mathrm{N}_{G}(\langle\varphi\rangle)\right|\left(\sum 1 / m_{i}+\sum 1 / n_{i j}\right),
$$

where $\mathrm{N}$ stands for the normalizer and the sums are taken respectively over canonical elliptic generators and consecutive canonical reflections for which $\varphi$ is conjugate to a power of $\theta\left(x_{i}\right)$ and $\theta\left(c_{i j-1} c_{i j}\right)$ respectively. 
Theorem 3.3. An involution $\sigma$ of $X$ has

$$
\sum[\mathrm{C}(G, \theta(c)): \theta(\mathrm{C}(\Lambda, c))]
$$

ovals, where $\mathrm{C}$ denotes the centralizer and the sum is taken over nonconjugate canonical reflections of $\Lambda$, whose images under $\theta$ are conjugate to $\sigma$ in $G$.

The proof of Theorem 3.2: If $\varphi$ has order $n$, then the number of its fixed points equals the number of the conjugacy classes of cyclic subgroups of order $n$ of $\Gamma_{\varphi}=\theta^{-1}(\langle\varphi\rangle)$. Clearly each such subgroup is generated by an elliptic element. However, each elliptic element is conjugate either to a power of some canonical elliptic generator or to a power of the product of two consecutive canonical reflections of $\Lambda$ and we say that fixed points of $\varphi$ are produced by $x_{i}$ or $c_{i j-1} c_{i j}$ in each of these cases respectively. We have to find how many fixed points of $\varphi$ is produced by each element $x_{i}$ and by each product $c_{i j-1} c_{i j}$.

Assume first, that $x_{i}$ produces fixed points of $\varphi$. Then, since conjugate elements have the same number of fixed points, by exchanging $\varphi$ with a suitable conjugation, we may assume that $x_{i}^{n_{i}} \in \Gamma_{\varphi}$ for $n_{i}=m_{i} / n$. Now $w x_{i}^{n_{i}} w^{-1} \in \Gamma_{\varphi}$ if and only if $w \in \theta^{-1}\left(\mathrm{~N}_{G}(\langle\varphi\rangle)\right)$. Observe however, that $w x_{i}^{n_{i}} w^{-1}$ and $w^{\prime} x_{i}^{n_{i}} w^{\prime-1}$ are conjugate in $\Gamma_{\varphi}$ if and only if $w^{-1} \gamma w^{\prime} \in$ $\mathrm{N}_{\Lambda}\left(\left\langle x_{i}^{n_{i}}\right\rangle\right)=\left\langle x_{i}\right\rangle$ for some $\gamma \in \Gamma_{\varphi}$, which means that $w^{-1} w^{\prime} \in\left\langle x_{i}\right\rangle \Gamma_{\varphi}$. Thus $x_{i}$ produces

$\left[\theta^{-1}\left(\mathrm{~N}_{G}(\langle\varphi\rangle)\right):\left\langle x_{i}\right\rangle \Gamma_{\varphi}\right]=\left[\theta^{-1}\left(\mathrm{~N}_{G}(\langle\varphi\rangle)\right) / \Gamma:\left\langle x_{i}\right\rangle \Gamma_{\varphi} / \Gamma\right]=\left|\mathrm{N}_{G}(\langle\varphi\rangle)\right| / m_{i}$

fixed points of $\varphi$.

Similarly, assume that $c_{i j-1} c_{i j}$ produces fixed points of $\varphi$. Then again we may assume that $\left(c_{i j-1} c_{i j}\right)^{m_{i j}} \in \Gamma_{\varphi}$ for $m_{i j}=n_{i j} / n$. Also $w\left(c_{i j-1} c_{i j}\right)^{m_{i j}} w^{-1} \in \Gamma_{\varphi}$ if and only if $w \in \theta^{-1}\left(\mathrm{~N}_{G}(\langle\varphi\rangle)\right)$. On the other hand $w\left(c_{i j-1} c_{i j}\right)^{m_{i j}} w^{-1}$ and $w^{\prime}\left(c_{i j-1} c_{i j}\right)^{m_{i j}} w^{\prime-1}$ are conjugate in $\Gamma_{\varphi}$ if and only if $w^{-1} \gamma w^{\prime}$ belongs to $\mathrm{N}_{\Lambda}\left(\left\langle\left(c_{i j-1} c_{i j}\right)^{m_{i j}}\right\rangle\right)=\left\langle c_{i j-1} c_{i j}\right\rangle$, for some $\gamma \in \Gamma_{\varphi}$, which means that $w^{-1} w^{\prime} \in\left\langle c_{i j-1} c_{i j}\right\rangle \Gamma_{\varphi}$. Hence $c_{i j-1} c_{i j}$ produces

$$
\begin{aligned}
{\left[\theta^{-1}\left(\mathrm{~N}_{G}(\langle\varphi\rangle)\right):\left\langle c_{i j-1} c_{i j}\right\rangle \Gamma_{\varphi}\right] } & =\left[\theta\left(\theta^{-1}\left(\mathrm{~N}_{G}(\langle\varphi\rangle)\right)\right): \theta\left(\left\langle c_{i j-1} c_{i j}\right\rangle \Gamma_{\varphi}\right)\right] \\
& =\left[\mathrm{N}_{G}(\langle\varphi\rangle):\left\langle\theta\left(c_{i j-1} c_{i j}\right)\right\rangle\right] \\
& =\left|\mathrm{N}_{G}(\langle\varphi\rangle)\right| / n_{i j}
\end{aligned}
$$

fixed points of $\varphi$. 
The proof of Theorem 3.3: The proof here is similar to the proof of the formula for the number of ovals of a symmetry of Riemann surface from [7]. We have to count reflections of $\Lambda$, which are in $\Gamma_{\sigma}=\theta^{-1}(\langle\sigma\rangle)$ but are not conjugate there. If $\operatorname{Fix}(\sigma)$ contains an oval, then $\sigma$ is conjugate to $\theta\left(c_{i}\right)$ for some canonical reflection $c_{i}$ of $\Lambda$. Now, without loss of generality, we may assume that $\theta\left(c_{i}\right)=\sigma$, since conjugate symmetries have the same number of ovals. Observe that for $w \in \Lambda, c_{i}^{w} \in \Gamma_{\sigma}$ if and only if $w \in \theta^{-1}\left(\mathrm{C}\left(G, \theta\left(c_{i}\right)\right)\right)$. Denote the last by $C_{i}$ and observe that it normalizes $\Gamma_{\sigma}$. Thus for $v, w \in C_{i}$, the reflections $c_{i}^{v}$ and $c_{i}^{w}$ of $\Gamma_{\sigma}$ are conjugate in $\Gamma_{\sigma}$ if and only if $w^{-1} v \in \mathrm{C}\left(\Lambda, c_{i}\right) \Gamma_{\sigma}$. As a consequence, conjugates of $c_{i}$ give rise to

$$
\left[C_{i}: \mathrm{C}\left(\Lambda, c_{i}\right) \Gamma_{\sigma}\right]=\left[\mathrm{C}\left(G, \theta\left(c_{i}\right)\right): \theta\left(\mathrm{C}\left(\Lambda, c_{i}\right)\right)\right]
$$

empty period cycles in $\Gamma_{\sigma}$.

Let now $c_{i^{\prime}}^{w} \in \Gamma_{\sigma}$ for some $i^{\prime} \neq i$ and $w \in \Lambda$. Then $\theta(w) \theta\left(c_{i^{\prime}}\right) \theta(w)^{-1}=$ $\sigma$ and so

$$
w C_{i^{\prime}} w^{-1}=C_{i} .
$$

Indeed, if $\lambda \in w C_{i^{\prime}} w^{-1}$ then $\theta(w)^{-1} \theta(\lambda) \theta(w) \in \mathrm{C}\left(G, \theta\left(c_{i^{\prime}}\right)\right)$. Thus $\theta(\lambda)$ centralizes $\sigma$ and so $\lambda \in C_{i}$. Conversely, if $\lambda \in C_{i}$, then $\theta(\lambda)$ normalizes $\sigma$. Hence $\theta(\lambda) \theta(w) \theta\left(c_{i^{\prime}}\right) \theta(w)^{-1} \theta(\lambda)^{-1}=\sigma$, which in turn means that $\theta(w)^{-1} \theta(\lambda) \theta(w)$ centralizes $\theta\left(c_{i^{\prime}}\right)$ and so $\lambda \in w C_{i^{\prime}} w^{-1}$ as claimed.

Furthermore, $c_{i^{\prime}}^{v} \in \Gamma_{\sigma}$ if and only if $v w^{-1} \in C_{i}$. Indeed, if this is the case, then $\theta(v) \theta\left(c_{i^{\prime}}\right) \theta(v)^{-1}=\sigma$ and so $\theta(w)^{-1} \theta(v) \in \mathrm{C}\left(\Lambda, \theta\left(c_{i^{\prime}}\right)\right)$. Thus $w^{-1} v \in C_{i^{\prime}}$, which gives $v w^{-1} \in w C_{i^{\prime}} w^{-1}=C_{i}$. The converse is similar and we omit it.

Finally, given $u, u^{\prime} \in C_{i}$ and $v=u w, v^{\prime}=u^{\prime} w$, the reflections $c_{i^{\prime}}^{v}, c_{i^{\prime}}^{v^{\prime}}$ are conjugate in $\Gamma_{\sigma}$ if and only if $v^{-1} v^{\prime} \in \mathrm{C}\left(\Lambda, c_{i^{\prime}}\right) w^{-1} \Gamma_{\sigma} w=\mathrm{C}\left(\Lambda, c_{i^{\prime}}\right) \Gamma$, which means that $u^{-1} u^{\prime} \in w \mathrm{C}\left(\Lambda, c_{i^{\prime}}\right) \Gamma w^{-1}$. Therefore, by (4), the conjugates of $c_{i^{\prime}}$ give rise to

$\left[C_{i}: w \mathrm{C}\left(\Lambda, c_{i^{\prime}}\right) \Gamma w^{-1}\right]=\left[C_{i^{\prime}}: \mathrm{C}\left(\Lambda, c_{i^{\prime}}\right) \Gamma\right]=\left[\mathrm{C}\left(\theta(\Lambda), \theta\left(c_{i^{\prime}}\right)\right): \theta\left(\mathrm{C}\left(\Lambda, c_{i^{\prime}}\right)\right)\right]$

empty period cycles in $\Gamma_{\sigma}$ and so the result follows.

Remark 3.4. The algebraic type of the centralizers of reflections was found by Singerman in his thesis [15] (see also [16]). However, what made Theorem 3.3 effective, is the fact that by going a bit more into details in the Singerman's papers, one can find explicit generators for these groups (e.g. $[\mathbf{2}],[\mathbf{9}]$ ). 


\section{Some examples}

We finish the paper by developing some examples in which we find topological type of the fixed-points sets for all automorphisms for some extremal actions of finite groups on non-orientable, unbordered Klein surfaces. Let $\nu(g)$ be the largest possible number of automorphisms of a non-orientable unbordered Klein surface of topological genus $g$. Then we have the following result due to Conder, Maclachlan, Todorovic Vasiljevic and Wilson.

Theorem $4.1([6])$. If $g$ is odd then $\nu(g) \geq 4 g$. If $g$ is even then $\nu(g) \geq 8(g-2)$. Furthermore, these bounds are sharp for infinitely many $g$.

Example 4.2. The action of order $4 g$ from Theorem 4.1 is given in [6] by the epimorphism

$$
\theta: \Lambda \rightarrow G=\mathrm{D}_{2 g}=\left\langle u, v \mid u^{2}, v^{2 g},(u v)^{2}\right\rangle,
$$

where $\Lambda$ is an NEC-group with signature $(0 ;+;[-] ;\{(2,2,2, g)\})$ and

$$
\theta\left(c_{0}\right)=u, \quad \theta\left(c_{1}\right)=u v^{g}, \quad \theta\left(c_{2}\right)=v^{g}, \quad \theta\left(c_{3}\right)=u v^{2} .
$$

Corollary 4.3. The following table gives the topological structures of the sets of points fixed by automorphisms acting on extremal Klein surfaces from Example 4.2

\begin{tabular}{|c|c|c|}
\hline $\begin{array}{c}\text { Representative } \\
\text { of a conjugacy class }\end{array}$ & $\begin{array}{c}\text { Isolated } \\
\text { fixed points }\end{array}$ & Ovals \\
\hline$u$ & 2 & 1 \\
$v^{g}$ & $2 g$ & 1 \\
$u v^{g}$ & 0 & 1 \\
$u v^{g+2}$ & 2 & 0 \\
$v^{2 \alpha}$ & 4 & 0 \\
\hline
\end{tabular}

Automorphisms from the remaining conjugacy classes have no fixed points.

Proof: Here $c_{i}, c_{i+1} \in \mathrm{C}\left(\Lambda, c_{i}\right)$ for $i=1,2$ and so $\theta\left(\mathrm{C}\left(\Lambda, c_{i}\right)\right)$ has at least 4 elements. Also, $c_{1}, c_{2}, c_{3} \in \mathrm{C}\left(\Lambda, c_{2}\right)$ and so $\theta\left(\mathrm{C}\left(\Lambda, c_{2}\right)\right)=\mathrm{D}_{2 g}$. So using our formulas and some obvious facts concerning normalizers and centralizers in dihedral groups, we obtain the above topological structure of the set of fixed points of all elements of $G$. 
Example 4.4. The case of even $g$ is technically more involved. Here, the action of order $8(g-2)$ was given in $[\mathbf{6}]$ in two steps. First, let $\Lambda$ be an NEC-group with signature $(0 ;+;[-] ;\{(2,2,2,4)\})$ and consider homomorphism

$$
\theta: \Lambda \rightarrow H=\mathrm{Z}_{g-2} \rtimes\left(\mathrm{D}_{4} \times \mathrm{Z}_{2}\right)=\langle w\rangle \rtimes\left(\left\langle u, v \mid u^{2}, v^{4},(u v)^{2}\right\rangle \times\langle t\rangle\right),
$$

given by

$$
\theta\left(c_{0}\right)=w u, \quad \theta\left(c_{1}\right)=v^{2}, \quad \theta\left(c_{2}\right)=t, \quad \theta\left(c_{3}\right)=u v,
$$

where each of the generators $u, v, t$ conjugates $w$ to its inverse. Then, the image $G$ of $\theta$ has order $8(g-2)$ and it acts on a non-orientable unbordered Klein surface of topological genus $g$.

Corollary 4.5. The following table gives the topological structures of the sets of points fixed by automorphisms acting on extremal Klein surfaces

\begin{tabular}{|c|c|c|}
\hline $\begin{array}{c}\text { Representative } \\
\text { of a conjugacy class }\end{array}$ & $\begin{array}{c}\text { Isolated } \\
\text { fixed points }\end{array}$ & Ovals \\
\hline$w u$ & 0 & 1 \\
\hline$v^{2}$ & $2(g-2)$ & 2 \\
\hline$t$ & 0 & 2 \\
\hline$u v$ & 0 & $g / 2-1$ \\
\hline$w u v^{2}$ & 4 & 0 \\
\hline$v^{2} t$ & 8 & 0 \\
\hline \multirow{2}{*}{ tuv } & $\int 4 g \equiv 0(4)$ & 0 \\
\hline & $8 \quad g \not \equiv 0(4)$ & \\
\hline$v w$ & 4 & 0 \\
\hline
\end{tabular}
from Example 4.4

Automorphisms from the remaining conjugacy classes have no fixed points.

Proof: Now,

$$
\begin{aligned}
& \mathrm{C}\left(\Lambda, c_{0}\right)=\left\langle c_{0}\right\rangle \oplus\left\langle c_{1}\right\rangle *\left\langle\left(c_{0} c_{3}\right)^{2}\right\rangle \\
& \mathrm{C}\left(\Lambda, c_{3}\right)=\left\langle c_{3}\right\rangle \oplus\left\langle c_{2}\right\rangle *\left\langle\left(c_{0} c_{3}\right)^{2}\right\rangle \\
& \mathrm{C}\left(\Lambda, c_{i}\right)=\left\langle c_{i}\right\rangle \oplus\left(\left\langle c_{i-1}\right\rangle *\left\langle c_{i+1}\right\rangle\right) \text { for } i=1,2
\end{aligned}
$$


by $[\mathbf{1 6}]$ (see also $[\mathbf{2}],[\mathbf{9}]$ ). Furthermore the images of $c_{0}, c_{1}, c_{2}, c_{3}$, $c_{1} c_{2}, c_{2} c_{3}, c_{0} c_{3}$ are pairwise non-conjugate in $G$ and straightforward, but rather tedious, calculations of their centralizers give the above results.

Acknowledgements. The author is deeply grateful to the referee for her/his inquiring questions, very accurate and helpful comments which allowed to improve initially submitted version of the paper a lot.

\section{References}

[1] N. L. Alling And N. Greenleaf, "Foundations of the theory of Klein surfaces", Lecture Notes in Mathematics 219, SpringerVerlag, Berlin-New York, 1971.

[2] E. Bujalance, F. J. Cirre, J. M. Gamboa, and G. GroMADZKI, On symmetries of compact Riemann surfaces with cyclic groups of automorphisms, J. Algebra 301(1) (2006), 82-95.

[3] E. Bujalance, A. F. Costa, S. M. Natanzon, and D. SingerMAN, Involutions of compact Klein surfaces, Math. Z. 211(3) (1992), 461-478.

[4] E. Bujalance, A. F. Costa, and D. Singerman, Application of Hoare's theorem to symmetries of Riemann surfaces, Ann. Acad. Sci. Fenn. Ser. A I Math. 18(2) (1993), 307-322.

[5] E. Bujalance, J. J. Etayo, J. M. Gamboa, and G. GroMADZKI, "Automorphism groups of compact bordered Klein surfaces. A combinatorial approach", Lecture Notes in Mathematics 1439, Springer-Verlag, Berlin, 1990.

[6] M. Conder, C. Maclachlan, S. Todorovic Vasiljevic, And S. Wilson, Bounds for the number of automorphisms of a compact non-orientable surface, J. London Math. Soc. (2) 68(1) (2003), 65-82.

[7] G. Gromadzki, Groups of automorphisms of compact Riemann and Klein surfaces, Habilitation Thesis, University of Bydgoszcz (1993).

[8] G. Gromadzki, On a Harnack-Natanzon theorem for the family of real forms of Riemann surfaces, J. Pure Appl. Algebra 121(3) (1997), 253-269.

[9] G. Gromadzki, Symmetries of Riemann surfaces from a combinatorial point of view, in: "Topics on Riemann surfaces and Fuchsian groups" (Madrid, 1998), London Math. Soc. Lecture Note Ser. 287, Cambridge Univ. Press, Cambridge, 2001, pp. 91-112. 
[10] A. Harnack, Ueber die Vieltheiligkeit der ebenen algebraischen Curven, Math. Ann. 10(2) (1876), 189-198.

[11] A. H. M. HoARE AND D. Singerman, The orientability of subgroups of plane groups, in: "Groups-St. Andrews 1981" (St. Andrews, 1981), London Math. Soc. Lecture Note Ser. 71, Cambridge Univ. Press, Cambridge, 1982, pp. 221-227.

[12] M. Izquierdo And D. Singerman, On the fixed-point set of automorphisms of non-orientable surfaces without boundary, in: "The Epstein birthday schrift", Geom. Topol. Monogr. 1, Geom. Topol. Publ., Coventry, 1998, pp. 295-301 (electronic).

[13] A. M. Macbeath, The classification of non-euclidean plane crystallographic groups, Canad. J. Math. 19 (1967), 1192-1205.

[14] A. M. Macbeath, Action of automorphisms of a compact Riemann surface on the first homology group, Bull. London Math. Soc. 5 (1973), 103-108.

[15] D. Singerman, Non-Euclidean crystallographic groups and Riemann surfaces, Ph. D. Thesis, University of Birmingham (1969).

[16] D. Singerman, On the structure of non-Euclidean crystallographic groups, Proc. Cambridge Philos. Soc. 76 (1974), 233-240.

[17] G. Weichold, Über symmetrische Riemanns'che Flächen und die Periodicitäsmoduln der zugehörin Abel'schen Normalintegrale erster Gattung, Zeitschrift für Math. und Phys. 28 (1883), 321-351.

[18] H. C. Wilkie, On non-Euclidean crystallographic groups, Math. Z. 91 (1966), 87-102.

Institute of Mathematics

University of Gdańsk

Wita Stwosza 57

80-952 Gdańsk

Poland

E-mail address: greggrom@math.univ.gda.pl

Primera versió rebuda el 30 de juliol de 2007, darrera versió rebuda el 14 d'abril de 2008. 